\title{
XXXVIII. On tungsten
}

\section{F. Wöhler}

To cite this article: F. Wöhler (1825) XXXVIII. On tungsten , Philosophical Magazine Series 1, 66:330, 263-269, DOI: 10.1080/14786442508673961

To link to this article: http://dx.doi.org/10.1080/14786442508673961

曲 Published online: 10 Aug 2009.

Submit your article to this journal 주다

Џll Article views: 2

Q View related articles $₫$ 


\section{On Tungsten. By F. WÖHLER*.}

B EFORE describing some combinations of tungsten, which in my opinion have not hitherto been known, I will indicate one of the processes I employed to procure pure tungstic acid. I had noticed that on heating a mixture of tungstate of potash and of hydrochlorate of ammonia, the tungstic acid is reduced into oxide by the hydrogen of the ammonia, which separates on the mass being dissolved in water. This property $I$ have applied in the preparation of tungstic acid in the following manner : The mixture of pulverized wolfram and carbonate of potash being melted together, the tungstate of potash is dissolved in water, and a sufficient quantity of hydrochlorate of ammonia added; the mass is then evaporated to dryness, and ignited and fused in a Hessian crucible till the sal ammoniac be entirely decomposed or evaporated. On dissolving the melted mass in hot water, a black and heavy powder is obtained, which is the oxide of tungsten. This is boiled with a weak solution of pure potash, for the purpose of carrying off a small portion of supertungstate of potash difficult of solution. When it is desired to have tungstic acid, this oxide is to be heated in an open crucible: it takes fire and burns vividly, changing into a yellow powder.

\section{Oxide of Tungsten.}

This oxide was discovered by M. Berzelius, who obtained it by heating tungstic acid in hydrogen gas. The acid at first turns perfectly blue, then gradually dark brown. As I employed for this experiment the crystalline tungstic acid, such as is obtained by the decomposition of crystallized tungstate of ammonia, I obtained a crystalline oxide of a lustre almost metallic, which after burnishing preserved this lustre, and had a dark copper-colour. It may also be obtained under the same form, but with a perfect metallic lustre, and of the most beantiful copper-colour, by bringing into contact a mixture of tungstic acid and zinc filings with diluted muriatic acid. The hydrogen at first turns the tungstic acid blue, and afterwards reduces it to brilliant laminæ of a copper-colour. The oxide thus obtained is only preserved under water; for by contact with atmospheric air it instantly turns blue, and soon changes completely into a yellow acid.

On comparing the character of this oxide of tungsten with the black powder obtained by the treatment of tungstate of potash with sal ammoniac, - which, as I have said, is oxide of

* From A nnales de Chimie et de Physique, tom. xxix. p. 43. tungsten, 
tungsten,-it might rather be presumed that this is the tungsten in its metallic state, especially when I observe, that this black body acquires a white metallic brilliancy, although a very deep one, when burnished. But the degree of increase of weight it acquires in combustion proves that it is oxide of tungsten, and not the metal itself. Heated in the air it burns long before it reddens; and according to many experiments, 100 parts always combine with 8 parts of oxygen (i. e. with the same quantity as the brcwn oxide) while passing into the acid state; whilst 100 parts of metallic tungsten require nearly 25 parts of oxygen in order to be converted into tungstic acid. It seems to me remarkable that the same combination should exhibit itself under such different forms. It is probable that the different state of the aggregation of its molecules is the cause of this phænomenon, as is also observed in native oxide of iron, cinnabar, \&c.

In preparing the brown oxide of tungsten by heating the acid in a current of hydrogen gas, another phænomenon is presented, which I cannot well explain. It is a fact that it is difficult to prepare pure tungstic acid when it once contains a fixed alkali. On using such an acid containing a little potash or soda, to prepare the brown oxide by the hydrogen, this oxide is never obtained, but always metallic tungsten,--a method by which this metal may also be easily procured. It is washed with pure potash, in order to dissolve the difficultly soluble tungstate with which it is mixed: it is then a metallic powder, rather white, very heavy, which, heated in the air, ignites, and of which 100 parts increase by nearly 25 parts.

\section{Combination of the Oxide of Tungsten roith Soda.}

On melting and igniting the neutral tungstate of soda in hydrogen gas, no action of the latter substance on the former is observed; but if the same experiment be made with supertungstate of soda, the surface of the mass soon assumes the colour and metallic brilliancy of copper, which is gradually communicated to the whole mass. On cooling, the colour becomes a gold-yellow; and if the mass be then treated with water, the neutral tungstate of soda is dissolved, and a heavy crystalline powder, of the colour and almost the metallic lustre of gold, is left. The best method of making the experiment is by using a glass tube which has a bulb in the middle, or by making the salt almost red-hot in a current of dry hydrogen gas. The mass is then boiled with water. The remaining powder is first digested in concentrated muriatic acid, in order to decompose the insoluble tungstate attached to it: it is then boiled with a solution of pure potash; and at last washed in 
water. It is necessary to employ all these precautions if the combination is to be obtained in its pure state.

The super-tungstate of soda may be prepared by adding tungstic acid to the neutral salt while melting, until the last portions will no longer dissolve.

The yellow metallic substance, which I shall show to be a combination of oxide of tungsten with soda, is crystallized in regular cubes, which are larger in proportion to the slowness of theoperation by which they have been formed. Sometimes cavities are found in the reduced saline matter, the sides of which are formed by an aggregation of small and very brilliant cubes. This combination has, as I said before, a perfect metallic brilliancy even when rubbed on paper; its colour scarcely differs from that of gold, and on looking at such a crystalline powder in the sun, it displays a beauty and a brilliancy possessed by few chemical preparations. Suspended as a fine powder in water, and looked at towards the sun, it is, like gold, transparent and of a green colour.

It is decomposed by no acid whatever, not even by boiling aqua-regia. Nothing but concentrated fluoric acid decomposes and dissolves it. The solutions of pure alkalies have no effect on it. Heated in contact with atmospheric air, it changes colour, softens, melts in a uniform manner; and around the substance submitted to the experiment is formed a white enamel which is soluble in water. By then adding an acid, tungstic acid is precipitated. But the whole mass of the combination is never converted into that enamel, - - there always remains a part in the middle which does not decompose.

This decomposition is not more complete even in oxygen gas, although it be accompanied by combustion. Here, too, the fusible mass which is produced, forms a stratum which hinders the action of the gas on the interior portions. In a vacuum the combination may be heated without decomposition. It appears therefore very probable that the fusible substance formed in this case is nothing else but tungstate of soda, and that this new body contains at least tungsten and sodium; but it was difficult to decide if those bodies were in the oxidated or in the metallic state. The property of not being decomposed by aqua-regia was opposed to its being regarded as an alloy of tungsten and sodium, and its metallic properties rendered it difficult to admit that this combination was oxidated.

As this combination is not decomposed by the most powerfulre-agents, I was obliged to seek for other means; and found that it was decomposed by chlorine,-but only on its being strongly heated in this gas; otherwise there would be no reason why it should not also be decomposed in aqua-regia.

Vol. 66. No.330. Oct. 1825.

L 1

Heated 
Heated in chlorine, a slight incandescence takes place ; chloride of tungsten which volatilizes, and of which I shall speak hereafter, is formed. The other product of the combustion is a greenish mass, which, treated with water, gives chloride of sodium crystallized in cubes. The insoluble green powder is a mixture of tungstic acid with a little oxide; but the quantity of the acid is much larger than that of the chloride and oxide together. The result therefore seems to be, that the combination contains oxygen, the whole quantity of which, before the action of the chlorine, was distributed so as to form soda and oxide of tungsten, and which, by the combination of the chlorine with the sodium, entirely united with the tungsten in order to form tungstic acid. I obtained exactly the same result in employing the chlorine in a perfectly dry state; and by avoiding the least mixture of atmospheric air with the chlorine, in such manner that the formation of the tungstic acid could proceed neither from the oxygen of the water nor from that of the atmospheric air, 0.873 grain of the combination, decomposed by chlorine, gave 0.157 grain of chloride of sodium $=0.089$ grain of soda ; consequently, 10.6 parts of soda in 100 of the combination. But as I expected to have observed that the half-fused state of the chloride of sodium prevented the complete action of the chlorine on the combination, I tried another manner of analysing this body; viz. by sulphur, - which entirely decomposes it. 0.487 grain of the combination were melted with pure sulphur in a closed crucible of porcelain. The resulting mass had the appearance of sulphuret of tungsten, and weighed 0.55 grain. No sulphuret of sodium could be discovered, either with water or muriatic acid. It was therefore treated with aqua-regia, which converted it into pure tungstic acid. The fluid was made to evaporate on the acid, and then the whole mass ignited. The tungstic acid, placed in a filter, was washed till all the sulphate of soda which had been formed was dissolved. The pure acid, dried and heated, weighed 0.45 grain. This quantity corresponds with 86.2 parts of oxide of tungsten in 100 parts of the combination : the remainder, therefore, is the quantity of soda $=13.8$ parts. It seems therefore that this combination is composed of,

$$
\begin{aligned}
& \text { Axide of tungs. By calculation. By experiment. }
\end{aligned}
$$

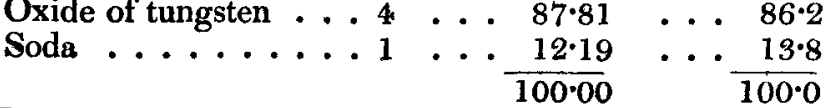

It will be seen that the oxygen of the soda is not half the quantity which is required to convert the oxide of tungsten into acid : for this purpose a part of the oxygen of the oxide 
of tungsten must be employed; and consequently a corresponding part of tungsten set free, and combined (as I have said before) with chlorine. From this reason it is evident that this new combination can only contain the tungsten in the state of oxide.

I have tried without success to produce this body by the direct combination of the oxide of tungsten with soda. By heating those bodies together I obtained metallic tungsten and tungstate of soda.

Lastly, I also tried to produce an analogous combination with potash, and treated the super-tungstate of potash in the same manner as the corresponding salt of soda. I obtained a metallic mass of a whitish colour, being pure metallic tungsten.

\section{Chloride of Tungsten.}

Sir H. Davy was the first who found that tungsten heated in chlorine burns and produces a white substance, which is decomposed by the action of water into tungstic acid and hydrochloric acid : but nothing further seems to be known on the subject. I have found that there are three different combinations of tungsten and chlorine.

\section{Perchloride of Tungsten.}

This is always produced, and nearly in a pure state, on heating the black oxide of tungsten in chlorine ;-with the brown oxide, tungstic acid is formed at the same time. The combination is effected with an evolution of light; the glass globe in which the operation is performed is filled with a thick yellow smoke, which is condensed into scales of a yellowish-black colour, and which at last forms a thick sublimate that exteriorly perfectly resembles native boracic acid. In contact with atmospheric air this chloride changes, according to the hygroscopic state of the air, within some hours or some days, into tungstic acid; and at the same time hydrochloric acid is disengaged. With water this decomposition is more rapid, although not instantaneous; very pure tungstic acid is deposited, and weak hydrochloric acid is formed at the same time. In ammonia it dissolves with a slight noise and an evolution of heat. It volatilizes in a low temperature without first melting, and its vapour is of a dark yellow colour.

Heated on platinum foil by a spirit-lamp, it is decomposed at the moment when it is volatilized, by the action of the aqueous vapour formed by the combustion of the alcohol: vapour of hydrochloric acid is formed; and the tungstic acid which is produced, forms above the flame a luminous smoke, and then disperses in large and very light flakes. 
The chloride of tungsten being decomposed in water into tungstic acid and hydrochloric acid, must correspond in its composition with the tungstic acid; - viz. it must be composed of 3 atoms of chlorine, and 1 of tungsten, thus :

$$
\begin{aligned}
& \text { Atoms. } \\
& \text { Chlorine . . . . . 35.9 } \\
& \text { Tungsten } \ldots 1 \ldots \frac{64 \cdot 1}{100 \cdot 0}
\end{aligned}
$$

By an approximative experiment, 0.166 grain of this chloride, dissolved in ammonia, waporated and heated, yielded 0.13 grain of tungstic acid $=62 \cdot 65$ parts of tungsten for 100 parts of the chloride.

\section{Protochloride of Tungsten.}

This compound is always formed, with a very slight mixture of the others, on heating metallic tungsten in chlorine. The metal ignites and is completely converted into chloride, which is presented sometimes under the form of an aggregation of fine tender needles of a deep red, resembling wool, but more frequently as a compact melted mass of a deep red colour, with a shining fracture nearly like that of cinnabar. It easily melts, and boils before it is volatilized. Its vapour has a red colour, deeper than that of nitrous acid. In water this chloride soon becomes violet, decomposing gradually and completely into oxide of a violet colour, and hydrochloric acid. This chloride is dissolved, with evolution of hydrogen gas, in a solution of pure potash; it produces tungstate of potash, and chloride of potassium. With caustic ammonia, hydrogen gas is also disengaged; but in this instance a yellowish solution is formed, which loses its colour: on being heated very slowly, brown oxide of tungsten is deposited.

This chloride seems to bear an analogy with the oxide, and must be composed of,

$$
\begin{aligned}
& \text { Atoms. } \\
& \text { Chlorine . . 2 . 26.79 } \\
& \text { Tungsten } \ldots 1 \ldots \frac{73 \cdot 21}{100.00}
\end{aligned}
$$

The third combination of chlorine with tungsten, - on the composition of which, however, I have made no experiment, and on which I shall make no conjecture,-is generally formed with the perchloride, although in very small quantity. I obtained it once in a larger quantity on heating sulphuret of tungsten in chlorine. This third chloride is the most beautiful of any : it is formed in transparent needles of a beautiful red colour, and often of great length; it melts very easily by a gentle heat, and crystallizes on cooling in long transparent 
M. Vauquelin on the Existence of Iodine in Minerals. 269

radii which are spread over the glass. It is more volatile than the other chlorides; its vapour has the colour of nitrous acid. In contact with the atmosphere it changes instantaneously into tungstic acid. Thrown into water it effervesces like quicklime, and heat is evolved; a particular noise is heard as in the slaking of lime, and in a moment it is entirely changed into tungstic acid.

XXXIX. On the Existence of Iodine in Minerals. By M. VAUQUELIN*.

TODINE has as yet been found only in a few vegetables and some sea mollusca. M. Cantu, professor of chemistry at Turin, however, has lately discovered it in the mineral water of Asti ; but no one to my knowledge has as yet found it in combination in minerals.

M. Jos. Tabary having sent me a few weeks ago some argentiferous minerals, - which he had partly bought of some of the native tribes of South America, and partly collected himself in the vicinity of Mexico, within a circle of 25 leagues from that city, -in order that I might ascertain for him the quantity of silver and gold (if the latter should be contained in it), I was so fortunate as to make the discovery which I shall have the honour to communicate to the Academy.

One of these minerals, called virgin silver of serpentine, and the physical properties of which are, $1 \mathrm{st}$, a whitish colour on its surface worn by friction, presenting grains of metallic silver; 2nd, a lamellar fracture of a yellowish-green colour, with some parts black and of metallic silver,-is that in which $I$ found the iodine.

Twenty grammes of this mineral treated with nitric acid effervesced, and evolved some nitrous gas towards the end of the operation. After having boiled it for some time, the liquid diluted with water presented two substances: the one very heavy, becoming quickly precipitated; the other, light, remaining a long time suspended in the fluid. They were separated from each other by decantation, washed, and dried.

The first, which weighed $6 \frac{42}{100}$ grammes, was easily melted before the blowpipe, producing a purplish flame; and after some time a globule of silver appeared in the midst of a melted substance, which spread over the charcoal like chloride of lead. The edges of the charcoal were invested with a yellow powder. The other substance, which was brown, weighed $2 \frac{70}{100}$

"From Annales de Chimie, tom. xxix. p. 99. 